\section{Sensibilisierung oder Toleranz - das ist hier die Frage}

\author{
Ob das Immunsystem nach einem Allergenkontakt mit Sensibili- \\ sierung oder mit Toleranz reagiert, hängt nach Ansicht von Prof. \\ Dr. Randolf Brehler maßgeblich von der Dosis ab. Wie er im folgen- \\ den Gespräch erläutert, hat das Konsequenzen für die spezifische \\ Immuntherapie ebenso wie für die Prävention allergischer \\ Erkrankungen.
}

? Herr Professor Brehler, nach Ihrer Überzeugung hat die Allergendosis einen entscheidenden Einfluss darauf, ob sich Sensibilisierung oder Toleranz ausbildet. Auf welche Erkenntnisse stützen Sie Ihre These?

Brehler: Auf der einen Seite wissen wir heute sehr genau, dass bei einer Hyposensibilisierung hohe Allergendosen Toleranz induzieren. Es gibt weiter gute Hinweise aus älteren Studien auf die Ausbildung einer Sensibilisierung durch sehr niedrige Allergendosen. Nach der damals gelegentlich üblichen Verwendung von Mischungen unterschiedlichster Allergene in einem Extrakt konnten manchmal IgE-vermittelte Sensibilisierungen gegen Allergenbestandteile nachgewiesen werden, gegen die zuvor keine Sensibilisierung bestanden hatte.

Auf der anderen Seite wird heute intensiv diskutiert, wie das Immunsystem im Kindesalter dazu kommt, auf einen Allergenkontakt mit einer Sensibilisierung oder einer Toleranzentwicklung zu reagieren. Es gibt zunehmend Hinweise darauf, dass höhere Allergendosen eher zur Toleranz und mittlere oder geringe Dosen eher zur Sensibilisierung führen.

? Gibt es Vorstellungen zum zugrunde liegenden Mechanismus?

Brehler: Abhängig von der Allergendosis werden unterschiedliche Zellen angesprochen. Entscheidend sind außerdem andere Faktoren, die die Immunantwort beeinflussen. So gab es Versuche mit Ratten, die eher atopisch - „IgE high responder" - oder eher nicht atopisch - „IgE low responder“ - reagierten. Wenn man diese Ratten nach der Geburt Allergenen aussetzte, induzierte

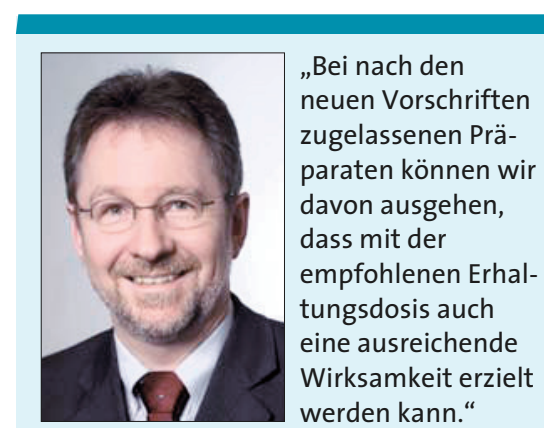

Prof. Dr. Randolf Brehler Klinik und Poliklinik für Hautkrankheiten, Universitätsklinikum Münster

man zunächst eine Stimulation von IgEAntikörpern. Bei den nicht atopischen Ratten verschwand das IgE bei weiterer Allergenexposition mit der Zeit wieder, es wurde Toleranz induziert. Bei den atopischen Ratten verstärkte sich die IgE-Reaktion hingegen durch weiteren Allergenkontakt. Auch diese Ratten konnten jedoch eine Toleranz entwickeln, wenn man die Allergendosis drastisch erhöhte. Demnach könnte die notwendige Dosis, die für die Toleranzentwicklung notwendig ist, je nach genetischem Hintergrund unterschiedlich sein.

? Welche Konsequenzen hat das für die subkutane und die sublinguale Immuntherapie?

Brehler: Die Allergendosis ist für die spezifische Immuntherapie von entscheidender Bedeutung. Wir wissen heute, dass sowohl die subkutane wie auch sublinguale Hyposensibilisierung als Hochdosistherapie erfolgen soll. Bei der subkutanen Therapie sollten während der Erhaltungstherapie pro Spritze etwa 5-20 $\mu$ g des Majorallergens verabreicht werden. Unter diesen Bedingungen ist nach heutiger Vorstellung eine gute Wirksamkeit bei akzeptablem Nebenwirkungsrisiko zu erzielen. Allerdings gibt es derzeit nur für wenige Allergene verbindliche Vorschläge für das exakte Verfahren zur Messung der Majorallergenkonzentration. Dennoch gilt allgemein, dass nur mit einer hohen Allergendosis eine optimale Wirkung zu erzielen ist.

Vor diesem Hintergrund wissen wir nicht so ganz genau, ob wir wirklich noch eine Toleranz induzieren oder nicht möglicherweise sogar die Sensibilisierung verstärken, wenn wir während der Hyposensibilisierung wegen Unverträglichkeit über lange Zeit sehr niedrige Dosen einsetzen. Zum Glück gibt es jedoch nur sehr wenige Patienten, die die vorgesehene Erhaltungsdosis nicht erreichen. Eine weitere Konsequenz ist das heute gültige Leitallergen-Prinzip: Durch Mischung unterschiedlicher Allergene wird die Dosis des einzelnen Allergens in der Mischung gesenkt und damit vermutlich die Wirksamkeit vermindert.

Bei der sublingualen Immuntherapie gibt es für ein Präparat eine sehr gute Dosisfindungsstudie, nach der es ganz klar eine optimale Dosis gibt: Auch hier ist eine hohe Dosis für eine gute Wirksamkeit nötig, wobei ein weiteres Steigern der Dosis den Effekt dann aber nicht mehr weiter vergrößert. Nach der Therapieallergene-Verordnung sind für alle neu zugelassenen Präparate Dosisfindungsstudien vorgeschrieben. $\mathrm{Zu}$ künftig können wir also davon ausgehen, dass zumindest bei den nach den neuen Vorschriften zugelassenen Präparaten mit der empfohlenen Erhaltungsdosis auch eine ausreichende Wirksamkeit erzielt werden kann. Die möglichen oralen Beschwerden unter der sublingualen Hochdosis-Immuntherapie lassen sich in der Regel gut mit Antihistaminika behandeln, die man vor allem in den ersten vier Wochen etwa 30 bis 60 Minuten vor Allergenapplikation geben kann.

? In einer kürzlich in Pediatric Allergy and Immunology publizierten Übersicht ${ }^{1}$ unterstreichen die Autoren, dass auch bei 